\title{
BioFeedback
}

\section{[Letter to the editor] Whole-genome amplification of sodium bisulfite-converted DNA can substantially impact quantitative methylation analysis using pyrosequencing}

Epigenetic gene silencing due to DNA hypermethylation plays an important role in the regulation of many cancer-related genes $(1,2)$. The investigation of DNA methylation patterns contributed to the understanding of various biological mechanisms in human cancer. As compared with previous methods (3-6), the pyrosequencing technique represents a highly sensitive method for quantification of DNA methylation $(7,8)$. All these approaches are based on sodium bisulfite conversion of genomic DNA (gDNA). However, analysis of DNA methylation in clinical specimens is restricted by limited availability of starting gDNA, bisulfite-dependent DNA degradation, and unstable cytosine-to-uracil transformation $(9,10)$. Therefore, whole-genome amplification (WGA) might represent a suitable method to overcome these limitations. One WGA method is the non-PCRbased isothermal multiple displacement amplification (MDA). In our study, we tested one of the commercially available kits for MDA-based WGA. The starting material was obtained from hematopoietic cells of 100 subjects, comprising patients with various hematological diseases as well as healthy volunteers. In order to assess the applicability to sodium bisulfite-converted
DNA, the DNA methylation of three genes $(C S X, p 15$, and $C A L R)$ was quantified at three different stages: after sodium bisulfite conversion (BC), after subsequent WGA (AMP), and after nucleic acid purification (SC) of the whole genome-amplified DNA. The evaluation comprised assessment of overall quality from pyrosequencing readings, comparison of the $\mathrm{CpG}$ methylation values before and after WGA, and data reproducibility (for details, see the Supplementary Material).

First, we observed a considerable reduction of the overall quality of the $C S X$ methylation analysis results for the WGA-treated samples AMP and SC as compared with the initial BC samples (Figure 1A). The percentages of passed $\mathrm{CpG}$ sites matching the pyrosequencing quality parameters for reliable methylation results were as follows: $79 \%$ of the BC CpGs, $19 \%$ of the AMP CpGs, and 36\% of the SC CpGs. Sixteen percent of the BC CpGs, $45 \%$ of the AMP CpGs, and $29 \%$ of the SC CpGs matched the quality criteria for check CpGs. Finally, 36\% of the analyzed CpG sites after WGA and 34\% after subsequent purification were detected as failed, while this failure occurred only in $6 \%$ of the $\mathrm{CpGs}$ in non-WGA-treated samples (for detailed information on pyrosequencing quality criteria, see the Supplementary Material). Furthermore, electrophoretic separation of PCR products revealed a specific band in BC-amplified samples and additional unspecific PCR fragments in reactions carried out with AMP or SC DNA input (Figure 1B), resulting in a high increase of signal quality warnings and divergent peak patterns in the pyrograms of WGA samples (Figure 1C).

The evaluation of absolute methylation values showed substantially different results after WGA. The differences were random and did not follow a specific pattern. We detected average differences in single CpG methylation of $11 \% \pm 11 \%$ (mean $\pm \mathrm{SD}$ ) in AMP and $10 \% \pm 11 \%$ in SC samples for $C S X, 6 \% \pm 9 \%$ in AMP and $6 \% \pm 8 \%$ in SC samples for $p 15$, and $3 \% \pm 0 \%$ in AMP and $3 \% \pm 5 \%$ in SC samples for CALR compared with BC specimens. For $C S X$, $42 \%$ of the total CpGs after WGA and $45 \%$ after subsequent purification showed a methylation difference of $>10 \%$ relative to the sodium bisulfite-converted samples (Figure 2A).

Reproducibility of the pyrosequencing results was determined using a set of 25 bisulfite-converted DNA samples and

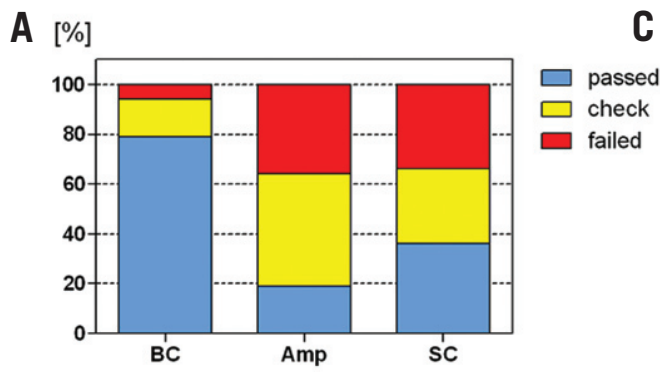

B

sample 1 sample 2 sample 3

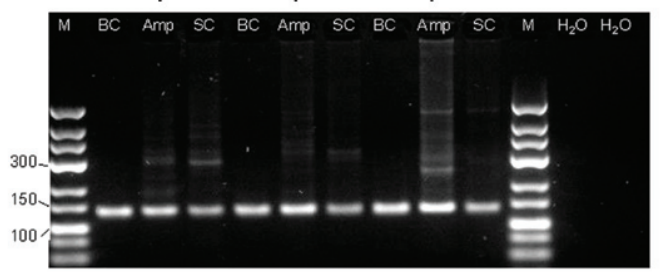

C
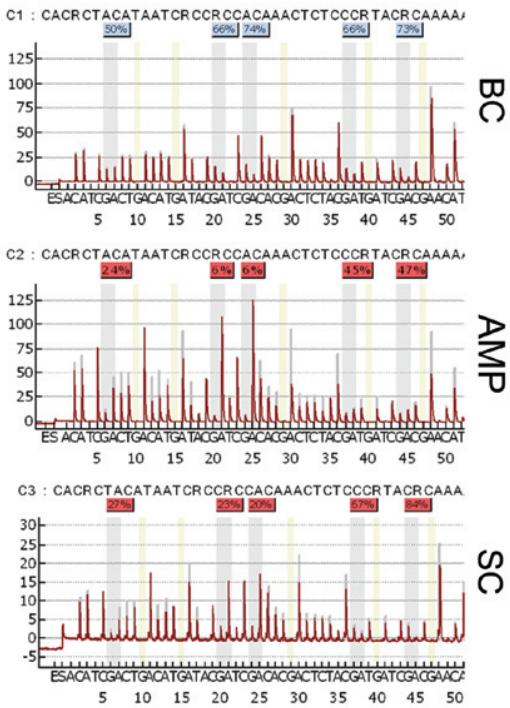

Figure 1. WGA leads to decreased signal quality of the pyrosequencing reactions. (A) The results of the CSX methylation analysis were utilized. The distributions of the different quality assessments of BC, AMP, and SC DNA are given in colored graph bars. Blue: passed if there were no failed or check quality assessments. Yellow: check if there were no failed. The trouble report comprised uncertainty due to low signalto-noise ratio (overall) and high CpG sum deviation, possible dispensation error at a specific dispensation position, and uncertain reference sequence pattern (overall) Red: failed due to low signal-to-noise ration (overall), not analyzed due to lack of data (overall) or failed bisulfite conversion at specific dispensation position. (B) Representative PCR products (samples 1-3) obtained from BC, AMP, and SC DNA samples. (C) Representative pyrograms of $B C, A M P$, and SC DNA samples illustrating the divergent peak pattern of the pyrosequencing-based methylation analysis results. 
A

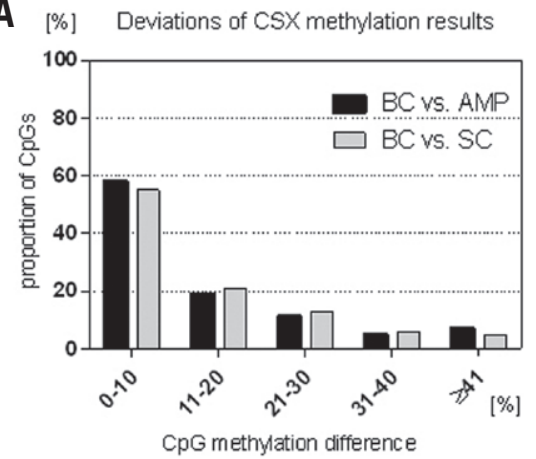

B

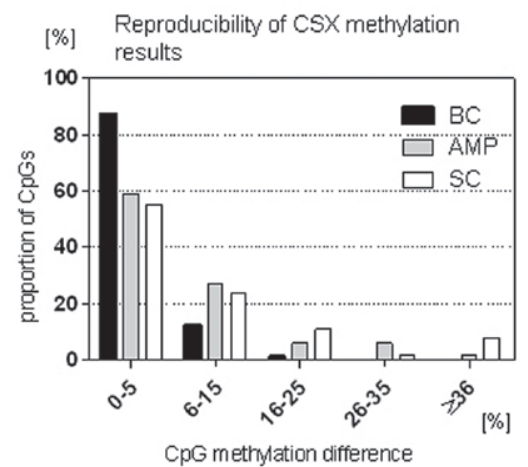

C

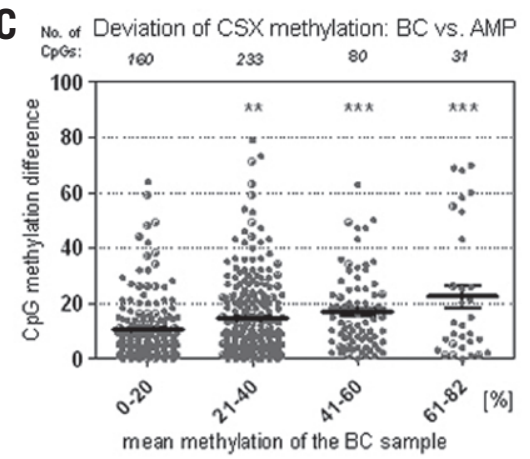

D

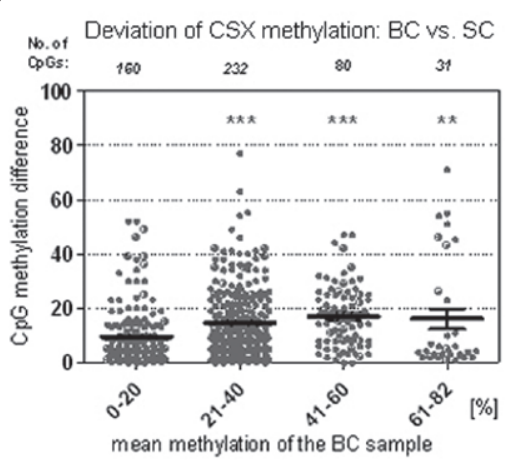

E No. of Deviation of $\mathrm{p} 15$ methylation: BC vs. AMP

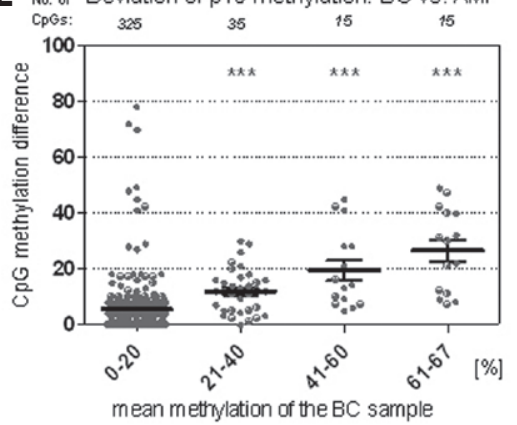

$\mathbf{F}$

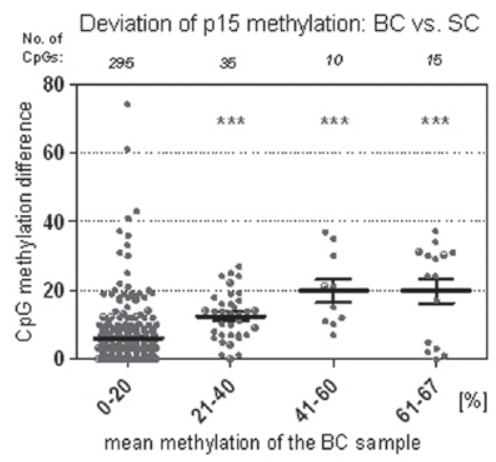

Figure 2. Decreased concordance and reproducibility of $\mathrm{CpG}$ methylation analysis results after WGA. (A) The graph bars display the deviations of the $C S X C p G$ methylation results generated by whole genome-amplified DNA (black bars) and purified DNA (gray bars) as compared with bisulfite-converted DNA without further modifications (BC versus AMP, BC versus SC). The deviations were graded into 5 ranks and were as follows: (0-10) $58 \%$ and $55 \%$, (11-20) $19 \%$ and $21 \%,(21-30) 11 \%$ and $13 \%,(31-40) 5 \%$ and $6 \%$, and $(\geq 41) 7 \%$ and $5 \%$. (B) The examination of the reproducibility of each CpG methylation data of the $C S X$ pyrosequencing was generated by bisulfite-converted DNA ( $B C$, black bars), bisulfite-converted, whole genome-amplified DNA ( $A M P$, gray bars) and SureClean purified DNA after WGA (SC, white bars) in independent, duplicate pyrosequencing experiments. The proportions of differences of the duplicate measurements for the bisulfite-converted samples, the whole genome-amplified samples, as well as the amplified and purified samples were as follows ( $B C$, AMP, SC): (0-5) 87\%, 59\%, and 55\%, (6-15) $12 \%, 27 \%$, and $24 \%,(16-25) 1 \%, 6 \%$, and $11 \%,(26-35) 0 \%, 6 \%$, and $2 \%$ as well as ( $\geq 36) 0 \%, 2 \%$, and $8 \%$. (C-F) The assessment of the CSX and p15 methylation showed that the CpG methylation values of the AMP samples after WGA and after subsequent nucleic-acid purification (SC) strongly deviated from their corresponding $B C$ samples depending on the mean sample methylation value of the $B C$ samples without WGA (graded into four ranks). Error bars indicate the standard error of the mean (SEM). ${ }^{*} P<0.05,{ }^{* *} P<0.01,{ }^{* * *} P<0.001$.

their corresponding WGA-treated samples in duplicate $\operatorname{CS} X$ pyrosequencing experiments. Methylation deviation of 5\% between single experiments was considered a threshold for reproducibility. While $87 \%$ of CpGs from bisulfite-converted samples were highly reproducible, the methylation values of $41 \%$ of the CpGs after WGA and $45 \%$ after subsequent purification deviated $>5 \%$ (Figure 2B), indicating a considerable decrease of reproducibility after WGA.

Additionally, our study showed that WGA caused a strong deviation of the methylation results of DNA targets, with initial methylation levels of $20-80 \%$ as detected for $p 15$ and CSX, representing a broad methylation range. WGA treatment of these $B C$ samples resulted in significantly increased $\mathrm{CpG}$ methylation differences of $16 \% \pm 15 \%$ in AMP and $16 \% \pm 14 \%$ in SC samples for $C S X$, as well as $17 \% \pm 13 \%$ in AMP and $16 \% \pm 10 \%$ in SC samples for $p 15$ (Figure 2, C-F). In contrast, WGA-treated samples of hypomethylated DNA $(<20 \%)$ exhibited significantly fewer deviations as observed in all three analyzed genes, specifically in the strongly hypomethylated $C A L R$ gene. A previous report compared DNA methylation profiles obtained from bisulfite-converted DNA treated with WGA to those without WGA treatment using the ABI Prism SNaPshot approach (10). The overall methylation difference was determined as $4 \% \pm 5 \%$. Of note, the vast majority of the analyzed samples in this study showed strong hypo- $(<20 \%)$ or hypermethylation $(>80 \%)$ in the non-WGA fraction. However, an exclusive analysis of the few samples harboring average methylation values of $20-80 \%$ revealed a mean CpG methylation difference of $13 \% \pm 5 \%$ between WGA and non-WGA specimens, indicating a trend toward higher methylation deviation in these samples. A further study reported the quantitative detection of DNA methylation by the qMAMBA technique after genome-wide amplification using a kit optimized for bisulfite-modified DNA (11). The authors observed a general agreement of methylation values in four genes before and after WGA. However, two of three genes exhibiting initial average methylation of $40-60 \%$ demonstrated distinct variations with differences of $\leq 25 \%$.

As noted above, additional unspecific PCR fragments in reactions carried out with $A M P$ or $S C D N A$ input might cause a high increase of signal quality warnings and divergent peak patterns in the corresponding pyrograms. Gel extraction and pyrosequencing of the specific PCR band resulted in improved methylation results of the WGA-treated samples (see Supplementary Figure $S 1$ and Supplementary Table S1). Therefore, inaccuracy of methylation results after the WGA step may be caused by unspecific sequencing primer binding due to additional PCR products presenting off target binding sites. Therefore, an optimization of the PCR reaction or gel extraction might contribute to an improvement of this method and must be evaluated in detail before application of WGA for pyrosequencing-based DNA methylation quantification.

In summary, pyrosequencing of three independent gene loci demonstrated a 
critical impact of WGA on quantitative DNA methylation when using a WGA kit not specifically optimized for bisulfite-converted DNA. This modification potentially leads to PCR interfering effects resulting in a considerable loss of pyrosequencing quality, impaired DNA methylation results and a decreased reproducibility. However, the suitability of this method depends on the experimental context. It still might be applicable for semiquantitative discrimination between very high or low DNA methylation, but not for exact quantification of small differences (which are frequently observed, for example, in clinical specimens to receive important diagnostic and prognostic informations). Therefore, we point out that the application of whole-genome amplification as a standard technique for subsequent quantitative DNA methylation analysis should be carefully evaluated with respect to the experimental studies planned.

\section{Acknowledgments}

We thank Ouidad Benlasfer for the technical support. Research in our laboratory was funded by the Gutermuth Foundation and the José Carreras Leukaemia Foundation. J.R. created and coordinated the project, performed sample preparation, pyrosequencing, data analysis, and wrote the paper. M.M. designed the pyrosequencing primer, established the pyrosequencing assay for CSX methylation analysis, assisted the data analysis, and assisted writing the paper. L.R. and A.K. performed sample preparation and pyrosequencing. D.H. assisted in creating the project and recruited patients. E.T.

\section{GE Healthcare}

Life Sciences

\section{More bands} for your buck

Now you can see more on every Western blot you do, because our new generation of detection reagent operates with superior levels of sensitivity, signal intensity and stability than Amersham ${ }^{T M} \mathrm{ECL}^{\text {TM }}$ Plus ever could. Whether you use film or a CCD imager, try it and see what your technique can really achieve.

Welcome to ECL Prime.

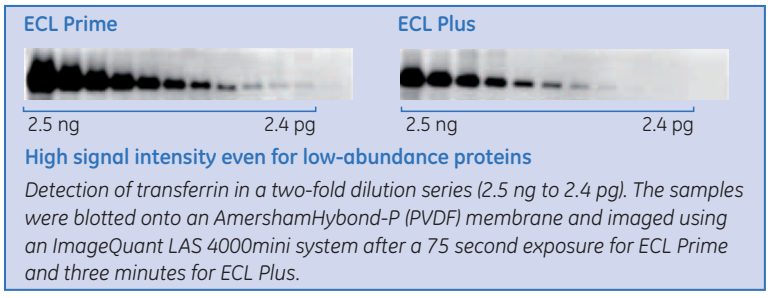

Find out more about Amersham ECL Prime at: www.gelifesciences.com/eclprime

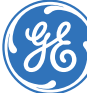

of GE Healthcare companies. enabled the research. W.K.H. coordinated the research, recruited patients, assisted the data analysis, assisted writing the paper, and provided funds.

\section{Competing interest statement}

The authors declare no competing interests.

\section{References}

1. Jones, P.A. and S.B. Baylin. 2007. The epigenomics of cancer. Cell 128:683692.

2.Herman, J.G. and S.B. Baylin. 2003. Gene silencing in cancer in association with promoter hypermethylation. N. Engl. J. Med. 349:20422054.

3. Clark, S.J., J. Harrison, C.L. Paul, and M. Frommer. 1994. High sensitivity mapping of methylated cytosines. Nucleic Acids Res. 22:2990-2997.

4.Herman, J.G., J.R. Graff, S. Myohanen, B.D. Nelkin, and S.B. Baylin. 1996. Methylation-specific PCR: a novel PCR assay for methylation status of $\mathrm{CpG}$ islands. Proc. Natl. Acad. Sci. USA 93:9821-9826.

5. Campan, M., D.J. Weisenberger, B. Trinh, and P.W. Laird. 2009. MethyLight. Methods Mol. Biol. 507:325-337.

6.Xiong, Z. and P.W. Laird. 1997. COBRA: a sensitive and quantitative DNA methylation assay. Nucleic Acids Res. 25:2532-2534.

7. Colella, S., L. Shen, K.A. Baggerly, J.P. Issa, and R. Krahe. 2003. Sensitive and quantitative universal pyrosequencing methylation analysis of $\mathrm{CpG}$ sites. BioTechniques 35:146-150.

8. Tost, J. and I.G. Gut. 2007. DNA methylation analysis by pyrosequencing. Nat. Protocols 2:2265-2275.

9. Mill, J. and A. Petronis. 2009. Profiling DNA methylation from small amounts of genomic DNA starting material: efficient sodium bisulfite conversion and subsequent whole-genome amplification. Methods Mol. Biol. 507:371-381.

10. Mill, J., S. Yazdanpanah, E. Guckel, S. Ziegler, Z. Kaminsky, and A. Petronis. 2006. Whole genome amplification of sodium bisulfitetreated DNA allows the accurate estimate of methylated cytosine density in limited DNA resources. BioTechniques 41:603-607.

11. Vaissiere, T., C. Cuenin, A. Paliwal, P. Vineis, G. Hoek, M. Krzyzanowski, L. Airoldi, A. Dunning, et al. 2009. Quantitative analysis of DNA methylation after whole bisulfitome amplification of a minute amount of DNA from body fluids. Epigenetics 4:221-230.

Received 24 June 2010; accepted 8 February 2011.

Jana Reins ${ }^{1}$, Maximilian Mossner ${ }^{3}$, Lennart Richter ${ }^{1}$, Anke Kmetsch ${ }^{1}$, Eckhard Thiel ${ }^{1}$, Detlef Haase $^{2}$, and Wolf-Karsten Hofmann ${ }^{3}$

${ }^{I}$ Department of Hematology and Oncology, Charité Campus Benjamin Franklin, Berlin, Germany, ${ }^{2}$ Department of Hematology and Oncology, University Hospital Göttingen, Germany, ${ }^{3}$ Department of Hematology and Oncology, University Hospital Mannheim, Germany

Address correspondence to Jana Reins, Charité University Hospital, Campus Benjamin Franklin, Department of Hematology, Oncology and Transfusion Medicine, Hindenburgdamm 30, Berlin, Germany.e-mail: jana.reins@charite.de

Supplementary material for this article is available at www.BioTechniques.com/article/113612. [ᄄi⿺𠃊

BioTechniques 50:161-164(March 2011) doi 10.2144/000113612

To purchase reprints of this article, contact: carmelitag@fosterprinting.com 